\title{
The bilinear formalism and the custodial symmetry in the two-Higgs-doublet model
}

\section{B. Grzadkowski, ${ }^{a}$ M. Maniatis ${ }^{b}$ and José Wudka ${ }^{c}$}

\author{
${ }^{a}$ Institute of Theoretical Physics, University of Warsaw, \\ Hoża 69, PL-00-681 Warsaw, Poland \\ ${ }^{b}$ Institute for Theoretical Physics, University of Bielefeld, \\ 33615 Bielefeld, Germany \\ ${ }^{c}$ Department of Physics, University of California, \\ Riverside CA 92521-0413, U.S.A. \\ E-mail: Bohdan.Grzadkowski@fuw.edu.pl, \\ Maniatis@physik.uni-bielefeld.de, jose.wudka@ucr.edu
}

ABSTRACT: We present a simple and transparent analysis of custodial symmetry in the Two-Higgs-Doublet Model adopting the bilinear formalism for the Higgs potential. The method allows to derive basis-independent, necessary and sufficient conditions for the potential to be invariant under custodial transformations. The relation between the custodial transformation and $\mathrm{CP}$ is revisited and clarified.

Keywords: Beyond Standard Model, Discrete and Finite Symmetries

ARXIV EPRINT: 1011.5228 


\section{Contents}

1 Introduction $\quad 1$

2 The custodial symmetry 3

3 Custodial transformations in terms of gauge invariant bilinears 4

$\begin{array}{lll}3.1 & \text { Equivalence of the two types of custodial transformations } & 7\end{array}$

$\begin{array}{ll}3.2 & \text { Basis independent conditions for CS }\end{array}$

4 Custodial transformations versus CP symmetry 9

5 Comments and conclusions $\quad 9$

\section{Introduction}

The $\rho$ parameter is experimentally measured as [1]

$$
\rho=1.0008_{-0.0007}^{+0.0017}
$$

where at tree-level $\rho \equiv m_{W}^{2} / \cos ^{2}\left(\theta_{W}\right) / m_{Z}^{2}$ with $\theta_{W}$ the Weinberg angle and $m_{W}$ and $m_{Z}$ the electroweak gauge boson masses.

In the Standard Model (SM) with a Higgs sector consisting of one Higgs doublet $\phi$ there is an extra symmetry of the Higgs potential

$$
V_{\mathrm{SM}}=-\lambda\left(\phi^{\dagger} \phi\right)+\mu\left(\phi^{\dagger} \phi\right)^{2} .
$$

which is responsible for $\rho \approx 1$; because of its role in insuring small corrections to $\rho$ this symmetry of $V_{\mathrm{SM}}$ is commonly called a custodial symmetry (CS) [2]. Its form can be made manifest by decomposing the complex Higgs doublet into the real components $\phi_{1}, \phi_{2}, \phi_{3}, \phi_{4}$,

$$
\phi=\left(\begin{array}{c}
\phi^{+} \\
\phi^{0}
\end{array}\right)=\left(\begin{array}{c}
\phi_{1}+i \phi_{2} \\
\phi_{3}+i \phi_{4}
\end{array}\right) .
$$

We then find immediately $\left(\phi^{\dagger} \phi\right)=\phi_{1}^{2}+\phi_{2}^{2}+\phi_{3}^{2}+\phi_{4}^{2}$, hence, the potential is invariant under $\mathrm{SO}(4) \sim \mathrm{SU}(2)_{L} \times \mathrm{SU}(2)_{R},(\sim$ means that both sides have the same Lie algebra $)$, and the $\mathrm{CS}$ is then the diagonal $\mathrm{SU}(2)$ subgroup.

In order to study the CS it is convenient to introduce the following matrix

$$
M \equiv\left(i \sigma^{2} \phi^{*}, \phi\right)=\left(\begin{array}{cc}
\phi^{0 *} & \phi^{+} \\
-\phi^{-} & \phi^{0}
\end{array}\right) .
$$


Then $M$ transforms under $\mathrm{SU}(2)_{L} \times \mathrm{SU}(2)_{R}$ as

$$
M \rightarrow L M R^{\dagger},
$$

where $L, R \in \mathrm{SU}(2)_{L, R}$ respectively. In the $\mathrm{SM}$ one can assume without loss of generality that the vacuum expectation value of $\phi$ is real, whence, after spontaneous symmetry breaking (SSB), $\langle M\rangle \propto \mathbb{1}_{2}$, so $\mathrm{SU}(2)_{L} \times \mathrm{SU}(2)_{R}$ is broken to $\mathrm{SU}(2)_{\text {diag }}$ (the diagonal subgroup) and it is the invariance of $\left|D_{\mu} \phi\right|^{2}$ with respect to $\mathrm{SU}(2)_{\text {diag }}$ that insures $\rho=1$ at tree-level $[2,3]$. The term custodial symmetry is reserved in the literature for the $\mathrm{SU}(2)_{\text {diag }}$ transformations under which both would-be Goldstone bosons and the corresponding gauge bosons transform as triplets. In this work we will also refer to custodial transformations as those generated by the full group $\mathrm{SU}(2)_{L} \times \mathrm{SU}(2)_{R}$. We will always assume that the vacuum respects the diagonal $\mathrm{SU}(2)_{\text {diag }}$, the $\mathrm{CS}$.

Now we can write

$$
\left(\phi^{\dagger} \phi\right)=\frac{1}{2} \operatorname{Tr}\left(M^{\dagger} M\right)
$$

which is manifestly invariant under (1.5) so that $V_{\mathrm{SM}}$ will be invariant under custodial transformations. It is easy to see, however, that the CS is explicitly violated in the SM by both the hypercharge gauge interactions and the Yukawa couplings; so the CS is but an approximate symmetry in the SM. Note that when the CS-violating coefficients are set to zero all massive vector bosons are mass degenerate (corresponding to $\rho=1$ ) to all orders in perturbation theory $[2,3]$.

It is easy to see that, even at tree-level, $\rho=1$ cannot be realized naturally in an extended scalar sector unless the scalar multiplets belong to a specific set of isospin representations. The singlets and isodoublets are the simplest of these " $\rho$-safe" representations; hereafter we will focus on isodoublet extensions of the SM. It is well known $[2,4-6]$ that even for two scalar isodoublets, in general, there exist potentially large radiative corrections to $\rho-1$ proportional to the squares of the scalar masses. A remedy has also been proposed $[2,4-6]$ through two generalizations of the custodial transformation to the two-Higgs-doublet model (THDM); in addition, it is worth noting that supersymmetric extensions of the standard model require a THDM which automatically respects a CS.

The potential relevance of CS breaking in the scalar sector of the THDM was nicely illustrated in [7], where it was used as a method to reconcile experimental constraints with a mass for the SM-like Higgs boson as high as $400-500 \mathrm{GeV}$, thereby ameliorating the hierarchy problem. This was accomplished by noting that the main experimental constraint on the SM Higgs-boson mass $m_{h}$ is derived from the $\rho$ parameter, which contains radiative contributions behaving as $\ln m_{h}$ for large $m_{h}$. In the presence of a second scalar doublet these logarithmic terms remain, but in addition the CS breaking terms generate contributions to $\rho$ that depend quadratically in the scalar masses, and compensate the logarithmic contributions.

In this note we will revisit this issue and derive basis-independent conditions for the CS for the THDM potential using both the conventional approach and the bilinear formalism of $[8,9]$, which allows to illustrate the CS in a transparent way; we also discuss the relation between custodial and CP invariance. In the calculations below the conventional approach 
is included only to confirm the results obtained within the bilinear formalism, the purpose of this short note is to illustrate the power of the latter. We will see that the bilinear formalism provides a very useful tool to discuss the potential of the THDM. It is, however, less suitable when considering the CS breaking effects generated by the U(1) couplings and fermion mass splittings. Within the conventional approach some basis-independent properties of the THDM have been previously discussed in the literature [10-13], and, more recently, also the CS [14].

\section{The custodial symmetry}

The most general potential for the THDM may be written in terms of the following doublets carrying the same hypercharge:

$$
\phi_{1}=\left(\begin{array}{c}
\phi_{1}^{+} \\
\phi_{1}^{0}
\end{array}\right), \quad \phi_{2}=\left(\begin{array}{c}
\phi_{2}^{+} \\
\phi_{2}^{0}
\end{array}\right) .
$$

Then the potential reads [15]

$$
\begin{aligned}
V= & m_{11}^{2}\left(\phi_{1}^{\dagger} \phi_{1}\right)+m_{22}^{2}\left(\phi_{2}^{\dagger} \phi_{2}\right) \\
& -m_{12}^{2}\left(\phi_{1}^{\dagger} \phi_{2}\right)-\left(m_{12}^{2}\right)^{*}\left(\phi_{2}^{\dagger} \phi_{1}\right) \\
& +\frac{1}{2} \lambda_{1}\left(\phi_{1}^{\dagger} \phi_{1}\right)^{2}+\frac{1}{2} \lambda_{2}\left(\phi_{2}^{\dagger} \phi_{2}\right)^{2}+\lambda_{3}\left(\phi_{1}^{\dagger} \phi_{1}\right)\left(\phi_{2}^{\dagger} \phi_{2}\right) \\
& +\lambda_{4}\left(\phi_{1}^{\dagger} \phi_{2}\right)\left(\phi_{2}^{\dagger} \phi_{1}\right)+\frac{1}{2}\left[\lambda_{5}\left(\phi_{1}^{\dagger} \phi_{2}\right)^{2}+\lambda_{5}^{*}\left(\phi_{2}^{\dagger} \phi_{1}\right)^{2}\right] \\
& +\left[\lambda_{6}\left(\phi_{1}^{\dagger} \phi_{2}\right)+\lambda_{6}^{*}\left(\phi_{2}^{\dagger} \phi_{1}\right)\right]\left(\phi_{1}^{\dagger} \phi_{1}\right) \\
& +\left[\lambda_{7}\left(\phi_{1}^{\dagger} \phi_{2}\right)+\lambda_{7}^{*}\left(\phi_{2}^{\dagger} \phi_{1}\right)\right]\left(\phi_{2}^{\dagger} \phi_{2}\right),
\end{aligned}
$$

with $m_{11}^{2}, m_{22}^{2}, \lambda_{1,2,3,4}$ real and $m_{12}^{2}, \lambda_{5,6,7}$ complex.

For studies of the CS within the THDM it is convenient to introduce the following set of matrices

$$
M_{i j} \equiv\left(\tilde{\phi}_{i}, \phi_{j}\right)=\left(\begin{array}{cc}
\phi_{i}^{0 \star} & \phi_{j}^{+} \\
-\phi_{i}^{-} & \phi_{j}^{0}
\end{array}\right),
$$

where $i=1,2$ refers to the scalar doublets. It is easy to see that all bilinears $\phi_{i}^{\dagger} \phi_{j}$ can be expressed in terms of $M_{11}$ and $M_{22}$, or in terms of $M_{12}$. Therefore the scalar potential (2.2) could also be written using $M_{11}$ and $M_{22}$ or $M_{12}$.

The following two versions of the custodial transformation for THDM have been considered in the literature [6]:

- Type I: In this case it is useful to express the potential in terms of $M_{11}$ and $M_{22}$. The transformation is a straightforward generalization of (1.5):

$$
M_{i i} \stackrel{C T_{I}}{\longrightarrow} M_{i i}^{\prime}=L M_{i i} R^{\dagger} \text { for } i=1,2
$$


- Type II: For this version of the custodial transformation, considered in [6], it is convenient to express the potential using $M_{12}$ only. The corresponding custodial transformation reads:

$$
M_{21} \stackrel{C T_{I I}}{\longrightarrow} M_{21}^{\prime}=L M_{21} R^{\dagger},
$$

where $L$ and $R$ belongs to $\mathrm{SU}(2)_{L}$ and $\mathrm{SU}(2)_{R}$, respectively.

Despite their different appearance, we will see that $C T_{I}$ and $C T_{I I}$ are equivalent under an appropriate field redefinition. Note however that for a fixed field basis one cannot simultaneously have invariance under (2.4) and (2.5) since, for example, the second case mixes $\phi_{1}$ and $\phi_{2}$ while the first one does not.

It is worth mentioning here that since both Higgs-boson doublets carry the same quantum numbers, physical content of the model we are considering cannot depend on a choice of the basis adopted for the scalar doublet fields $\left(\phi_{1}, \phi_{2}\right)$. Nevertheless, the form of the Lagrangian obviously changes by a change of basis; also the form of custodial transformation will change if we change the basis. In what follows we will investigate consequences of such a unitary basis transformation:

$$
\phi_{i} \rightarrow \hat{\phi}_{i}=\sum_{j} U_{i j} \phi_{j} \text { for } i=1,2
$$

where $U \in \mathrm{U}(2)$. This rotation implies the following change for $M_{i j}$

$$
M_{i j} \equiv\left(\tilde{\phi}_{i}, \phi_{j}\right) \rightarrow \hat{M}_{i j} \equiv\left(\hat{\tilde{\phi}}_{i}, \hat{\phi}_{j}\right)=\left(\sum_{k} U_{i k}^{\star} \tilde{\phi}_{k}, \sum_{l} U_{j l} \phi_{l}\right)=\frac{1}{2} \sum_{k l} M_{k l}\left(\begin{array}{cc}
U_{i k}^{\star} & 0 \\
0 & U_{j l}
\end{array}\right) \text {. }
$$

Note that above the sum stands in front of a product of matrices, so that the elements of summed matrices are correlated. From (2.7) we can determine the form of the custodial transformation in the new basis; for example, for type I (2.4) we obtain,

$$
\hat{M}_{i j} \stackrel{C T_{I}}{\longrightarrow} \frac{1}{2} \sum_{k} \sum_{o p} L \hat{M}_{o p}\left[\left(\begin{array}{cc}
U_{o k} & 0 \\
0 & U_{p k}^{\star}
\end{array}\right) R^{\dagger}\left(\begin{array}{cc}
U_{i k}^{\star} & 0 \\
0 & U_{j k}
\end{array}\right)\right] .
$$

\section{Custodial transformations in terms of gauge invariant bilinears}

Due to gauge invariance the doublets in the potential (2.2) can only appear in bilinear form, that is, in terms of $\left(\phi_{i}^{\dagger} \phi_{j}\right)$. It turns out that it is very convenient to discuss CS using the bilinears instead of the fields themselves. There are just four independent bilinears that can be combined as follows $[8,16]$

$$
K_{0}=\phi_{1}^{\dagger} \phi_{1}+\phi_{2}^{\dagger} \phi_{2}, \quad \mathbf{K}=\left(\begin{array}{c}
\phi_{1}^{\dagger} \phi_{2}+\phi_{2}^{\dagger} \phi_{1} \\
i \phi_{2}^{\dagger} \phi_{1}-i \phi_{1}^{\dagger} \phi_{2} \\
\phi_{1}^{\dagger} \phi_{1}-\phi_{2}^{\dagger} \phi_{2}
\end{array}\right)
$$

Any choice of $K_{0} \geq 0$ and $\mathbf{K}^{2} \leq K_{0}^{2}$ fix the doublets modulo a gauge transformation. The potential (2.2) can be expressed in terms of $K_{0}$ and $\mathbf{K}$ in a very compact and suggestive way

$$
V=\xi_{0} K_{0}+\boldsymbol{\xi}^{\mathrm{T}} \mathbf{K}+\eta_{00} K_{0}^{2}+2 K_{0} \boldsymbol{\eta}^{\mathrm{T}} \mathbf{K}+\mathbf{K}^{\mathrm{T}} E \mathbf{K}
$$


with the following real parameters

$$
\begin{array}{r}
\xi_{0}, \quad \eta_{00}, \quad \boldsymbol{\xi}=\left(\begin{array}{l}
\xi_{1} \\
\xi_{2} \\
\xi_{3}
\end{array}\right), \quad \boldsymbol{\eta}=\left(\begin{array}{l}
\eta_{1} \\
\eta_{2} \\
\eta_{3}
\end{array}\right), \\
E=E^{\mathrm{T}}=\left(\begin{array}{lll}
E_{11} & E_{12} & E_{13} \\
E_{12} & E_{22} & E_{23} \\
E_{13} & E_{23} & E_{33}
\end{array}\right)
\end{array}
$$

which have the following expressions in terms of the original parameters in (2.2):

$$
\begin{gathered}
\xi_{0}=\frac{1}{2}\left(m_{11}^{2}+m_{22}^{2}\right), \quad \boldsymbol{\xi}=\frac{1}{2}\left(\begin{array}{c}
-2 \operatorname{Re}\left(m_{12}^{2}\right) \\
2 \operatorname{Im}\left(m_{12}^{2}\right) \\
m_{11}^{2}-m_{22}^{2}
\end{array}\right), \\
\eta_{00}=\frac{1}{8}\left(\lambda_{1}+\lambda_{2}\right)+\frac{1}{4} \lambda_{3}, \quad \boldsymbol{\eta}=\frac{1}{4}\left(\begin{array}{c}
\operatorname{Re}\left(\lambda_{6}+\lambda_{7}\right) \\
-\operatorname{Im}\left(\lambda_{6}+\lambda_{7}\right) \\
\frac{1}{2}\left(\lambda_{1}-\lambda_{2}\right)
\end{array}\right), \\
E=\frac{1}{4}\left(\begin{array}{ccc}
\lambda_{4}+\operatorname{Re}\left(\lambda_{5}\right) & -\operatorname{Im}\left(\lambda_{5}\right) & \operatorname{Re}\left(\lambda_{6}-\lambda_{7}\right) \\
-\operatorname{Im}\left(\lambda_{5}\right) & \lambda_{4}-\operatorname{Re}\left(\lambda_{5}\right) & -\operatorname{Im}\left(\lambda_{6}-\lambda_{7}\right) \\
\operatorname{Re}\left(\lambda_{6}-\lambda_{7}\right) & -\operatorname{Im}\left(\lambda_{6}-\lambda_{7}\right) & \frac{1}{2}\left(\lambda_{1}+\lambda_{2}\right)-\lambda_{3}
\end{array}\right) .
\end{gathered}
$$

It is easy to see that the transformations of $K_{0}$ and $\mathbf{K}$ under a change of basis (2.6) read

$$
\begin{aligned}
K_{0} & \rightarrow K_{0}^{\prime}=K_{0}, \\
\mathbf{K} & \rightarrow \mathbf{K}^{\prime}=R(U) \mathbf{K},
\end{aligned}
$$

where the matrix $R(U) \in \mathrm{SO}(3)$ is given in terms of $U$ through

$$
U^{\dagger} \sigma^{a} U=R_{a b}(U) \sigma^{b}
$$

The effect of a change of basis (3.5) corresponds to the following change of the potential parameters

$$
\begin{aligned}
\boldsymbol{\xi} & \rightarrow \boldsymbol{\xi}^{\prime}=R(U) \boldsymbol{\xi} \\
\boldsymbol{\eta} \rightarrow \boldsymbol{\eta}^{\prime} & =R(U) \boldsymbol{\eta} \\
E \rightarrow E^{\prime} & =R(U) E R(U)^{\mathrm{T}} .
\end{aligned}
$$

Now we turn to the description of the CS in terms of these parameters.

Custodial transformation of type I. For the custodial transformation of type I (2.4) it is convenient to express $K_{0}, \mathbf{K}$ in terms of $M_{i i}$ with $i=1,2$ :

$$
\begin{aligned}
K_{0} & =\frac{1}{2} \operatorname{Tr}\left(M_{11}^{\dagger} M_{11}+M_{22}^{\dagger} M_{22}\right), \\
K_{1} & =\operatorname{Tr}\left(M_{11}^{\dagger} M_{22}\right), \\
K_{2} & =(-i) \operatorname{Tr}\left(M_{11} \tau_{3} M_{22}^{\dagger}\right), \\
K_{3} & =\frac{1}{2} \operatorname{Tr}\left(M_{11}^{\dagger} M_{11}-M_{22}^{\dagger} M_{22}\right) .
\end{aligned}
$$


whence the type I custodial transformation corresponds to

$$
\begin{aligned}
\mathrm{CT}_{I}: \quad K_{0,1,3} & \rightarrow K_{0,1,3}, \\
K_{2} & \rightarrow(-i) \operatorname{Tr}\left[M_{11}\left(R^{\dagger} \tau_{3} R\right) M_{22}^{\dagger}\right] .
\end{aligned}
$$

The invariance of the potential (3.2) under (3.9) restricts its parameters as follows

$$
\boldsymbol{\eta}=\left(\begin{array}{l}
\cdot \\
0 \\
\cdot
\end{array}\right), \quad \boldsymbol{\xi}=\left(\begin{array}{l}
\cdot \\
0 \\
\cdot
\end{array}\right), \quad E=\left(\begin{array}{ccc}
\cdot & 0 & \cdot \\
0 & 0 & 0 \\
\cdot & 0 & \cdot
\end{array}\right),
$$

where the dots denote arbitrary entries. The THDM will be symmetric under this custodial transformation if and only if there exists a basis rotation after which the potential parameters take the form (3.10).

Using (3.4) we can express the potential (3.2) with parameters (3.10) in terms of the original doublets:

$$
\begin{aligned}
V= & m_{11}^{2}\left(\varphi_{1}^{\dagger} \varphi_{1}\right)+m_{22}^{2}\left(\varphi_{2}^{\dagger} \varphi_{2}\right) \\
& -\operatorname{Re}\left(m_{12}^{2}\right)\left[\left(\varphi_{1}^{\dagger} \varphi_{2}\right)+\left(\varphi_{2}^{\dagger} \varphi_{1}\right)\right] \\
& +\frac{1}{4}\left(\lambda_{1}+\lambda_{2}\right)\left[\left(\varphi_{1}^{\dagger} \varphi_{1}\right)^{2}+\left(\varphi_{2}^{\dagger} \varphi_{2}\right)^{2}\right]+\lambda_{3}\left(\varphi_{1}^{\dagger} \varphi_{1}\right)\left(\varphi_{2}^{\dagger} \varphi_{2}\right) \\
& +\frac{1}{2}\left(\lambda_{4}+\operatorname{Re}\left(\lambda_{5}\right)\right)\left[\left(\varphi_{1}^{\dagger} \varphi_{2}\right)+\left(\varphi_{2}^{\dagger} \varphi_{1}\right)\right]^{2} \\
& +\operatorname{Re}\left(\lambda_{6}\right)\left[\left(\varphi_{1}^{\dagger} \varphi_{2}\right)+\left(\varphi_{2}^{\dagger} \varphi_{1}\right)\right]\left(\varphi_{1}^{\dagger} \varphi_{1}\right) \\
& +\operatorname{Re}\left(\lambda_{7}\right)\left[\left(\varphi_{1}^{\dagger} \varphi_{2}\right)+\left(\varphi_{2}^{\dagger} \varphi_{1}\right)\right]\left(\varphi_{2}^{\dagger} \varphi_{2}\right)
\end{aligned}
$$

This potential is invariant under the custodial transformation of type I and matches the expression in [6].

Custodial transformation of type II. In this case it is useful to express $K_{0}$ and $\mathbf{K}$ in terms of $M_{21}$ only:

$$
\begin{aligned}
& K_{0}=\operatorname{Tr}\left(M_{21}^{\dagger} M_{21}\right), \\
& K_{1}=2 \operatorname{Re}\left(\operatorname{det} M_{21}^{\dagger}\right), \\
& K_{2}=-2 \mathbf{I m}\left(\operatorname{det} M_{21}\right), \\
& K_{3}=-\operatorname{Tr}\left(M_{21} \tau_{3} M_{21}^{\dagger}\right)
\end{aligned}
$$

which then transform as follows:

$$
\begin{aligned}
\mathrm{CT}_{I I}: \quad K_{0,1,2} & \rightarrow K_{0,1,2} \\
K_{3} & \rightarrow-\operatorname{Tr}\left[M_{21}\left(R^{\dagger} \tau_{3} R\right) M_{21}^{\dagger}\right] .
\end{aligned}
$$

It follows that in order for the potential to be invariant under this custodial transformation the parameters take the form

$$
\boldsymbol{\eta}=\left(\begin{array}{c}
\cdot \\
\cdot \\
0
\end{array}\right), \quad \boldsymbol{\xi}=\left(\begin{array}{c}
\cdot \\
\cdot \\
0
\end{array}\right), \quad E=\left(\begin{array}{ccc}
\cdot & \cdot & 0 \\
\cdot & \cdot & 0 \\
0 & 0 & 0
\end{array}\right)
$$


Again we can use (3.4) to write the potential in terms of the two doublets as presented in [6]:

$$
\begin{aligned}
V= & \frac{1}{2}\left(m_{11}^{2}+m_{22}^{2}\right)\left[\left(\varphi_{1}^{\dagger} \varphi_{1}\right)+\left(\varphi_{2}^{\dagger} \varphi_{2}\right)\right] \\
& -m_{12}^{2}\left(\varphi_{1}^{\dagger} \varphi_{2}\right)-\left(m_{12}^{2}\right)^{*}\left(\varphi_{2}^{\dagger} \varphi_{1}\right) \\
& +\frac{1}{4}\left(\lambda_{1}+\lambda_{2}+2 \lambda_{3}\right)\left[\left(\varphi_{1}^{\dagger} \varphi_{1}\right)+\left(\varphi_{2}^{\dagger} \varphi_{2}\right)\right]^{2} \\
& +\lambda_{4}\left(\varphi_{1}^{\dagger} \varphi_{2}\right)\left(\varphi_{2}^{\dagger} \varphi_{1}\right)+\frac{1}{2}\left[\lambda_{5}\left(\varphi_{1}^{\dagger} \varphi_{2}\right)^{2}+\lambda_{5}^{*}\left(\varphi_{2}^{\dagger} \varphi_{1}\right)^{2}\right] \\
& +\left(\operatorname{Im}\left(\lambda_{6}\right)+\operatorname{Im}\left(\lambda_{7}\right)\right) i\left[\left(\varphi_{1}^{\dagger} \varphi_{2}\right)-\left(\varphi_{2}^{\dagger} \varphi_{1}\right)\right]\left(\varphi_{1}^{\dagger} \varphi_{1}\right) \\
& +\left(\operatorname{Im}\left(\lambda_{6}\right)+\operatorname{Im}\left(\lambda_{7}\right)\right) i\left[\left(\varphi_{1}^{\dagger} \varphi_{2}\right)-\left(\varphi_{2}^{\dagger} \varphi_{1}\right)\right]\left(\varphi_{2}^{\dagger} \varphi_{2}\right) .
\end{aligned}
$$

Note that the parameters are in general complex in this case.

Here we immediately see the advantage of the bilinear formalism: while the potentials in conventional notation, (3.11) and (3.15) look quite different, in terms of bilinears $K_{0} \mathbf{K}$ they are very similar (compare (3.10) with (3.14)). In the next section we will show that both potentials are related by a simple basis transformation.

\subsection{Equivalence of the two types of custodial transformations}

Here we will show that type I (2.4) and type II (2.5) custodial transformation are equivalent, that is, these are the same transformations expressed in different bases.

In the bilinear formalism this is evident: the parameters (3.10) and (3.14), corresponding to the type I and II custodial transformation are related by a change of basis (3.7) with

$$
\begin{aligned}
& R_{I \rightarrow I I}^{(1)}=\left(\begin{array}{ccc}
\sin \alpha & 0 & -\cos \alpha \\
\cos \alpha & 0 & \sin \alpha \\
0 & -1 & 0
\end{array}\right) \quad \text { or } \\
& R_{I \rightarrow I I}^{(2)}=\left(\begin{array}{ccc}
\sin \alpha & 0 & \cos \alpha \\
\cos \alpha & 0 & -\sin \alpha \\
0 & 1 & 0
\end{array}\right)
\end{aligned}
$$

Then solving the equation (3.6) one finds that the corresponding $U$ is

$$
U_{I \rightarrow I I}=\frac{e^{i \gamma}}{\sqrt{2}}\left(\begin{array}{c}
e^{-i \varphi} \pm i e^{-i \varphi} \\
e^{+i \varphi} \mp i e^{+i \varphi}
\end{array}\right)
$$

where $\gamma$ is an undetermined phase (the method involving bilinears is not sensitive to an overall phase, which can always be absorbed by a hypercharge transformation of the doublets) and upper and lower signs correspond to $R_{I \rightarrow I I}^{(1)}$ with $\varphi=-\alpha / 2+\pi / 2$, and $R_{I \rightarrow I I}^{(2)}$ with $\varphi=-\alpha / 2$, respectively. The rotation $U_{I \rightarrow I I}$ can, of course, also be obtained from (2.8) by requiring that the $\hat{\phi}_{i}$ transform according to (2.5) whenever the $\phi_{i}$ transform according to $(2.4)$.

We close this section with a comment on the equivalence of these two types of custodial transformation in a complete theory that also contains fermions. For the clarity of the argument, let us first assume that Yukawa interactions are absent. Then consider the 
following two versions (I and II) of THDM: I) A model with the potential $V_{I}(\vec{\phi})$ and II) a model with the potential $V_{I I}(\vec{\phi}):=V_{I}(U \vec{\phi})$ (equivalent to $V_{I}(\vec{\phi})$, just written in a different basis); the potentials (3.11) and (3.15) serve as an illustration of these two versions, as they are related by the unitary transformation (3.17). As long as Yukawa interactions are not present the two models are physically identical, they differ only by a field redefinition. Now let us switch on Yukawa interactions of the same form in both Lagrangians, $\vec{\phi} \bar{\psi} \vec{\Gamma}_{Y} \psi$, so that $\mathcal{L}_{I}(\vec{\phi})=\cdots-V_{I}(\vec{\phi})+\vec{\phi} \bar{\psi} \vec{\Gamma}_{Y} \psi+\cdots$ while $\mathcal{L}_{I I}(\vec{\phi})=\cdots-V_{I}(U \vec{\phi})+\vec{\phi} \bar{\psi} \vec{\Gamma}_{Y} \psi+\cdots$. Obviously, now $\mathcal{L}_{I}$ and $\mathcal{L}_{I I}$ are no longer equivalent; they differ by the potentials. However it is interesting to realize that where the difference between them is located is a matter of basis choice: the basis change $\vec{\phi} \rightarrow U^{-1} \vec{\phi}$ performed upon $\mathcal{L}_{I I}$ would shift the difference from the potentials to the Yukawa interactions. It is then interesting to note that in the perturbative expansion only those processes are sensitive to the difference between the two versions that incorporate both Yukawa couplings and couplings that emerge from scalar potentials (so e.g. scalar masses). For instance, the vector-boson vacuum polarizations would be exactly the same in both models at 1-loop (but not in higher orders).

The equivalence (by a basis transformation) between the two types of the transformations that we have found above clearly shows a need for a basis independent formulation of an invariance under the custodial transformation. That issue is discussed in the next section.

\subsection{Basis independent conditions for CS}

The two types of custodial transformations considered above are related by a change of basis and are therefore equivalent, but it would clearly be desirable to have a basis-independent set of conditions which insure that a scalar potential is invariant under custodial transformations. In terms of the bilinear coefficients in (3.2) these conditions are the following

$$
\begin{aligned}
E . \mathbf{v} & =0 \\
\boldsymbol{\xi} \cdot \mathbf{v}=\boldsymbol{\eta} \cdot \mathbf{v} & =0
\end{aligned}
$$

for some $\mathbf{v} \neq 0$. In order to prove the assertion we note that these conditions are basis independent since the first one is equivalent to requiring $\operatorname{det}(E)=0$. This means that if the conditions (3.18) are satisfied in one basis, they are satisfied in any basis. Therefore it is sufficient to show that (3.18) are necessary and sufficient conditions for a custodial transformation in a specific basis, for instance the one defined by the parameters (3.14).

First we have to show that (3.14) imply (3.18), which is immediate: $\mathbf{v}=(0,0,1)$ is the zero eigenvector of $E$ in (3.14), and $\mathbf{v}$ is indeed orthogonal to $\boldsymbol{\xi}, \boldsymbol{\eta}$. Now, assume (3.18), then, since $E$ is symmetric and has one zero eigenvalue, we can choose a basis where $E=\operatorname{diag}\left(E_{1}, E_{2}, 0\right)$, so that we can take $\mathbf{v}=(0,0,1)$, and this will be orthogonal to $\boldsymbol{\xi}, \boldsymbol{\eta}$ only if both these vectors take the form $(\cdot, \cdot, 0)$; it follows that there is a basis where (3.18) imply (3.14). 


\section{Custodial transformations versus CP symmetry}

The relation between the custodial transformation and $\mathrm{CP}$ was first noticed in [6]. In this section we re-derive these results using the bilinear formalism as an illustration of the usefulness of this parameterization in discussing both symmetries. First we recall the CP transformation of the doublets, which is defined by

$$
\mathrm{CP}: \quad \varphi_{i}(x) \rightarrow \varphi_{i}^{*}\left(x^{\prime}\right), \quad i=1,2 .
$$

Here we have explicitly written the argument of the fields, since the argument is changed under the CP transformation, that is, we have $x^{\prime}=\left(x^{0},-\mathbf{x}\right)$. Applying (4.1) to the bilinears (3.1) we see that a CP transformation is a reflection on the 1-3 plane - in addition to the parity transformation for the field argument [16]:

$$
\begin{aligned}
\mathrm{CP}: \quad K_{0,1,3}(x) & \rightarrow K_{0,1,3}\left(x^{\prime}\right), \\
K_{2}(x) & \rightarrow-K_{2}\left(x^{\prime}\right)
\end{aligned}
$$

We recognize that like in the type I of the custodial transformation (3.9) only the bilinear $K_{2}$ transforms non trivially under CP. We can now easily give the Higgs potential, invariant under (4.2), which has to have the following parameters [16]

$$
\boldsymbol{\xi}=\left(\begin{array}{l}
\cdot \\
0 \\
.
\end{array}\right), \quad \boldsymbol{\eta}=\left(\begin{array}{l}
\cdot \\
0 \\
\cdot
\end{array}\right), \quad E=\left(\begin{array}{ccc}
\cdot & 0 & \cdot \\
0 & \cdot & 0 \\
\cdot & 0 & \cdot
\end{array}\right) \text {. }
$$

By a comparison with (3.10) we find that the only difference is the central entry of the matrix $E$. We thus can state that any Higgs potential, invariant under custodial symmetry, is automatically invariant under the CP transformation. Note that the opposite is in general not true. This result holds in any basis, since we can for any custodial symmetric model by a change of basis - go to the parameterization (3.10). We can also find this result by a comparison of the basis-independent conditions given for the CP transformation in [16] and for the custodial symmetry given in (3.18).

It is worth noticing that the requirement of breaking $\mathrm{SU}(2)_{L} \times \mathrm{SU}(2)_{R}$ down to $\mathrm{SU}(2)_{\text {diag }}$ implies $v_{i}=v_{i}^{\star}$, in other words the possibility of spontaneous CP violation is also eliminated by the requirement of invariance under custodial transformations.

\section{Comments and conclusions}

The custodial symmetry in the SM is respected by the Higgs potential implying no corrections to the $\rho$ parameter which grow as $\propto m_{h}^{2}$. However in the Two-Higgs-Doublet Model the potential in general does not respect this symmetry. In this paper, employing the bilinear formalism, we have formulated basis independent conditions (3.18) which allow for an easy verification of the custodial symmetry of a potential. Yukawa and U(1) interactions break the custodial symmetry explicitly and generate potentially large radiative corrections to the $\rho$ parameter. Phenomenologically this results in significant constraints on the 
parameters of the model, such as the allowed spitting among scalar or fermion-doublet masses, see for example, [17].

We have also shown that the two types of custodial transformations in the THDM discussed in the literature are related by a field redefinition and can be distinguished physically only through the Yukawa interactions.

We have also clarified relations between the custodial symmetry and CP; it has been shown that any potential which is symmetric under custodial transformations is also invariant under $\mathrm{CP}$.

Shortly after this paper appeared so did another study [14], where equivalent basisindependent conditions for CS were found using a different approach. In later works, the custodial [18] and other symmetries [19] of $N$-Higgs-doublet models have been investigated using the bilinear formalism.

\section{Acknowledgments}

M. M. wants to thank O. Nachtmann for very helpful discussions. This work is supported in part by the Ministry of Science and Higher Education (Poland) as research project N N202 006334 (2008-11). The work of J. W. is supported in part by the U. S. Dept. of Energy Grant No. DE-FG03-94ER40837.

Open Access. This article is distributed under the terms of the Creative Commons Attribution Noncommercial License which permits any noncommercial use, distribution, and reproduction in any medium, provided the original author(s) and source are credited.

\section{References}

[1] Particle Data Group collaboration, K. Nakamura et al., Review of particle physics, J. Phys. G 37 (2010) 075021 [inSPIRE].

[2] P. Sikivie, L. Susskind, M.B. Voloshin and V.I. Zakharov, Isospin breaking in technicolor models, Nucl. Phys. B 173 (1980) 189 [inSPIRE].

[3] M.E. Peskin and D.V. Schroeder, An introduction to quantum field theory, Addison-Wesley, Reading U.S.A. (1995) [INSPIRE].

[4] D. Toussaint, Renormalization effects from superheavy Higgs particles, Phys. Rev. D 18 (1978) 1626 [INSPIRE].

[5] R. Lytel, Weak isospin breaking and higher order corrections, Phys. Rev. D 22 (1980) 505 [INSPIRE].

[6] A. Pomarol and R. Vega, Constraints on CP-violation in the Higgs sector from the $\rho$ parameter, Nucl. Phys. B 413 (1994) 3 [hep-ph/9305272] [INSPIRE].

[7] R. Barbieri, L.J. Hall and V.S. Rychkov, Improved naturalness with a heavy Higgs: an alternative road to LHC physics, Phys. Rev. D 74 (2006) 015007 [hep-ph/0603188] [INSPIRE].

[8] F. Nagel, New aspects of gauge-boson couplings and the Higgs sector, Ph.D. thesis, Fakultät für Physik und Astronomie, Institut für Theoretische Physik, Ruperto-Carola University of Heidelberg, Heidelberg Germany (2004) [INSPIRE]. 
[9] M. Maniatis, A. von Manteuffel, O. Nachtmann and F. Nagel, Stability and symmetry breaking in the general two-Higgs-doublet model, Eur. Phys. J. C 48 (2006) 805 [hep-ph/0605184] [INSPIRE].

[10] S. Davidson and H.E. Haber, Basis-independent methods for the two-Higgs-doublet model, Phys. Rev. D 72 (2005) 035004 [Erratum ibid. D 72 (2005) 099902] [hep-ph/0504050] [INSPIRE].

[11] H.E. Haber and D. O'Neil, Basis-independent methods for the two-Higgs-doublet model. II. The significance of $\tan \beta$, Phys. Rev. D 74 (2006) 015018 [hep-ph/0602242] [INSPIRE].

[12] C. Nishi, Physical parameters and basis transformations in the two-Higgs-doublet model, Phys. Rev. D 77 (2008) 055009 [arXiv:0712.4260] [INSPIRE].

[13] P. Ferreira, H.E. Haber and J.P. Silva, Basis invariant conditions for supersymmetry in the two-Higgs-doublet model, Phys. Rev. D 82 (2010) 016001 [arXiv:1004.3292] [InSPIRE].

[14] H.E. Haber and D. O'Neil, Basis-independent methods for the two-Higgs-doublet model III: the CP-conserving limit, custodial symmetry and the oblique parameters $S, T, U$, Phys. Rev. D 83 (2011) 055017 [arXiv: 1011.6188] [INSPIRE].

[15] H.E. Haber and R. Hempfling, The renormalization group improved Higgs sector of the minimal supersymmetric model, Phys. Rev. D 48 (1993) 4280 [hep-ph/9307201] [InSPIRE].

[16] M. Maniatis, A. von Manteuffel and O. Nachtmann, CP violation in the general two-Higgs-doublet model: a geometric view, Eur. Phys. J. C 57 (2008) 719 [arXiv: 0707.3344] [INSPIRE].

[17] H.-J. He, N. Polonsky and S.-F. Su, Extra families, Higgs spectrum and oblique corrections, Phys. Rev. D 64 (2001) 053004 [hep-ph/0102144] [INSPIRE].

[18] C. Nishi, Custodial $\mathrm{SO}(4)$ symmetry and CP-violation in N-Higgs-doublet potentials, Phys. Rev. D 83 (2011) 095005 [arXiv: 1103.0252] [INSPIRE].

[19] K. Olaussen, P. Osland and M. Solberg, Symmetry and mass degeneration in multi-Higgs-doublet models, JHEP 07 (2011) 020 [arXiv: 1007.1424] [INSPIRE]. 